\title{
Serial Ingestion or Co-ingestion of Creatine and Sodium Bicarbonate on Performance Following a Resistance Exercise Workout: A Pilot Study
}

\author{
By Theocharis Ispoglou* \\ David Legget" \\ James Driscoll ${ }^{\ddagger}$ \\ Kyriaki Kaldaridou ${ }^{+}$
}

To examine the ergogenic potential of creatine $(\mathrm{Cr})$, sodium bicarbonate $(\mathrm{SB})$, and $\mathrm{Cr}+\mathrm{SB}$ after completion of a resistance exercise bout (REB). Methods: Following recruitment, 27 trained males $(26.8 \pm 5.7$ y) completed a one repetition maximum (1RM) strength test in the parallel squat $(120.9 \pm 28.2 \mathrm{~kg})$. Participants then followed a standardised meal plan for four days whilst ingesting one of four supplements, and on day five they undertook the REB. This was a double blind randomised placebocontrolled study where participants ingested one of the following: Placebo (PLA, $\mathrm{n}=7), \mathrm{Cr}\left(20 \mathrm{~g} \mathrm{~d}^{-1}\right.$ and PLA, $\left.\mathrm{n}=8\right), \mathrm{SB}\left(0.5 \mathrm{~g} \mathrm{~kg}^{-1} \mathrm{~d}^{-1}\right.$ and PLA, $\left.\mathrm{n}=6\right)$, and $\mathrm{Cr}+\mathrm{SB}\left(5 \mathrm{~g} \mathrm{~d}^{-1}\right.$ of $\mathrm{Cr}$ and $0.5 \mathrm{~g} \mathrm{~kg}^{-1} \mathrm{~d}^{-1} \mathrm{SB}, \mathrm{n}=6$ ) divided in four doses. The REB consisted of $4 \times 10$ repetitions ( $70 \%$ of $1 \mathrm{RM}, 1 \frac{1}{2}$ min recovery). The primary performance outcome was a fifth set (70\% of 1RM) performed to volitional exhaustion. Blood glucose and lactate, heart rate, ratings of perceived exertion, sickness and stomachache scales, and time to complete each set were also recorded. Results: Participants in $\mathrm{Cr}, \mathrm{SB}$, and $\mathrm{Cr}+\mathrm{SB}$ completed the required number of repetitions at $70 \%$ of 1RM however in PLA they completed the same repetitions at lower intensity $(68+3.4 \%)$. Participants in PLA, Cr, $\mathrm{SB}$, and $\mathrm{Cr}+\mathrm{SB}$ completed $8 \pm 1.6,11 \pm 5.5,9 \pm 3.7$, and $11 \pm 3.3$ repetitions respectively during the fifth set. Despite the fact that no significant differences were observed in performance, small (SB: 0.4) and moderate effect (Cr: 0.7; $\mathrm{Cr}+\mathrm{SB}: 1.0)$ sizes were observed in relation to PLA. Body mass increased significantly after $\mathrm{Cr}(78.1 \pm 8.9 \mathrm{~kg}$ pre vs $79.1 \pm 8.9 \mathrm{~kg}$ post, $\mathrm{p}<0.05)$. No other significant differences existed in recorded variables. Conclusions: Performance gains were of higher magnitude in the $\mathrm{Cr}+\mathrm{SB}$ and $\mathrm{Cr}$ groups. Co-ingestion of a small amount of $\mathrm{Cr}\left(5 \mathrm{~g} \mathrm{~d}^{-1}\right)$ with a standard dose of SB $\left(0.5 \mathrm{~g} \mathrm{~kg}^{-1} \cdot \mathrm{d}^{-1}\right)$ appears to be equally beneficial as ingestion of a standard dose of $\mathrm{Cr}$ $\left(20 \mathrm{~g} \mathrm{~d}^{-1}\right)$. The $\mathrm{Cr}+\mathrm{SB}$ was not associated with significant gains in body mass which may be beneficial in sports where size gains may negate performance. Further research is required, with larger sample size, and specific athletic populations in order to confirm the findings of the current study.

\footnotetext{
${ }^{*}$ Principal Lecturer, Leeds Beckett University, UK.

${ }^{\dagger}$ Leeds Beckett University, UK.

"Leeds Beckett University, UK.

${ }^{+}$Leeds Beckett University, UK.
} 


\section{Introduction}

Athletes taking part in competitive sports are always seeking ways to improve their performance primarily through means of optimising training adaptations. Strategies that may enhance training capacity may range from sound nutrition practices, use of appropriate sport-specific equipment, adequate rest and sleep to use of nutritional supplements or ergogenic aids (Burke, 2007).

Resistance exercise training involving use of external resistance such as free weights is a widely used method employed by athletes as means to enhance muscle growth and ultimately strength (Baechle and Earle, 2008; Schoenfeld, 2010; Schoenfeld, 2013; Winwood et al., 2011; Andersen and Aagaard, 2010; Ahtiainen et al., 2003). In particular, hypertrophy type workouts characterised by high training volumes (3-6 sets of 8-12 repetitions), short recovery between sets (30-90 s) and medium intensities ( $70 \%$ of $1 \mathrm{RM})$ are considered to be of the most effective programmes for muscle growth gains, whilst they are also accompanied by high metabolic responses (Schoenfeld, 2010; Schoenfeld, 2013; Cairns, 2006; Nicholson et al., 2014). The repeated stimulation of skeletal muscle and reliance on glycolysis during these types of workouts results in hydrogen ion $(\mathrm{H}+)$ accumulation and metabolic acidosis, which are contributing factors to fatigue and performance decrements due to interference with the contractile processes and impairments in energy production (Kraemer et al., 1998; McCaulley et al., 2009; Nicholson et al., 2014). Therefore, any attempts to enhance the buffering capacity of both the skeletal muscle and blood will ameliorate the deleterious effects of muscle acidosis, and this will potentially enhance training capacity through reductions in fatigue levels. Ultimately, enhanced training capacity can result in greater gains in muscle growth and strength performance since there is a positive relationship between the cross sectional area of muscle and strength (Goldberg et al., 1975; Schoenfeld, 2010; Moritani and deVries, 1979).

It is also widely accepted that one of the training adaptations of hypertrophy type resistance workouts and other types of high intensity exercise is improved buffering capacity when these are routinely performed over a longer training period (Sharp et al., 1986; Parkhouse and McKenzie, 1984; Sahlin, 2014; Schoenfeld, 2010). Similarly to training, ergogenic aids such as creatine monohydrate and sodium bicarbonate may further enhance the buffering capacity of skeletal muscle and blood above and beyond training alone. The abovementioned intracellular (creatine) and extracellular buffers (sodium bicarbonate) are widely used ergogenic practices employed by athletes in sports relying primarily on glycolysis as the main source of energy production (Burke and Pyne, 2007; McNaughton et al., 2008; Douroudos et al., 2006). Supplementation with creatine monohydrate results in increased concentration of phosphocreatine stores which in turn results in increased rephosphorylation rates of ADP during high intensity exercise, and in the process, H+ are utilised and buffered (Hultman and Sahlin, 1980; Sahlin, 2014). On the other hand, bicarbonate loading results in elevations of $\mathrm{pH}$ and 
bicarbonate ions resulting in increased $\mathrm{H}+$ and lactate efflux from working muscle since $\mathrm{H}+$ are transported against their concentration gradient (Requena et al., 2005; Sahlin, 2014).

A typical creatine loading regime involves daily supplementation with 20 $\mathrm{g}$ of creatine for a period of five to seven days (main phase) followed by a longer supplementation period (maintenance phase) of two to three grams per day (Sahlin, 2014; Hultman et al., 1996). However, one of the disadvantages of the main phase of creatine loading is water retention and a subsequent increase in body mass (Greenhaff et al., 1994; Sahlin, 2014) which may be detrimental in sports where an increase in the power-to-body mass ratio is considered essential for successful performance. Therefore, alternative modes of supplementation protocols involving ingestion of smaller amounts of creatine may be more appropriate in a number of sports and for different training or competition conditions. The typical modes of bicarbonate loading involve the supplementation of either $0.3 \mathrm{~g} \mathrm{~kg}^{-1}$ of sodium bicarbonate $1-1 \frac{1}{1 / 2} \mathrm{~h}$ before exercise (acute loading) or ingestion of $0.5 \mathrm{~g} \mathrm{~kg}^{-1} \cdot \mathrm{d}^{-1}$ of sodium bicarbonate over a period of four to five days (serial loading). One of the advantages of serial over acute loading is that gastrointestinal discomfort may be avoided since smaller amounts of sodium bicarbonate (e.g. $0.125 \mathrm{~g} \mathrm{~kg}^{-1} \mathrm{x}$ four times daily) are given over a longer period (Burke and Pyne, 2007); while the benefits associated with increases in blood $\mathrm{pH}$ and bicarbonate ions are similar to acute dosages of $0.3 \mathrm{~g} \mathrm{~kg}^{-1}$ (McNaughton et al., 1999).

Despite the abundance of research studies on either creatine or sodium bicarbonate there is limited research examining the effectiveness of coingestion of both as ergogenic aids in particular during resistance training activities. It has been previously demonstrated that co-ingestion of both supplements may be advantageous during high intensity activities (Barber et al., 2013; Mero et al., 2004) however not all of these studies included a creatine and sodium bicarbonate alone conditions whilst the ingestion protocols involved either acute ingestion or short serial supplementation period (two days). A more recent study (Griffen et al., 2015) investigated the effects of creatine and sodium bicarbonate co-ingestion on mechanical power during high intensity exercise however there was no apparent additive effect of combining these two supplements together when compared to creatine. This was perhaps due to the fact that the sodium bicarbonate dosage $\left(0.3 \mathrm{~g} \mathrm{~kg}^{-1} \mathrm{~d}^{-1}\right)$ was lower than what would be perceived beneficial when serial loading is adopted or due to inadequate washout-period between the creatine containing conditions. No studies have tested the hypothesis that by combining a standard dose $\left(0.5 \mathrm{~g} \mathrm{~kg}^{-}\right.$ $\left.{ }^{1} \cdot \mathrm{d}^{-1}\right)$ of sodium bicarbonate with a small amount of creatine $\left(5 \mathrm{~g} \mathrm{~kg}^{-1} \cdot \mathrm{d}^{-1}\right)$ may further enhance training capacity and performance than a standard creatine dosage $\left(20 \mathrm{~g} \mathrm{~kg}^{-1} \mathrm{~d}^{-1}\right)$ or standard sodium bicarbonate dosage. Therefore, our main aim was to investigate whether combining these two buffers may be of greater benefit than the optimum supplementation protocols for each supplement alone. 


\section{Methodology}

\section{Participants}

Twenty-seven resistance trained male participants $(26.8 \pm 5.7 \quad \mathrm{y})$ volunteered and met the criteria for participation to this study. All participants had engaged in structured resistance training programmes for at least three months before the commencement of the study, they were technically competent in the back squat exercise, and they were free from illness and musculoskeletal injuries. Participants were excluded from the study if they were vegetarians or vegans since it has been shown that vegetarians have lower muscle creatine stores than omnivores (Maccormick et al., 2004; Burke et al., 2003; Shomrat et al., 2000). Participants refrained from taking any form of performance enhancing substances and supplements including creatine and sodium bicarbonate for at least four weeks before the onset of the study. This was to avoid any carryover effects of creatine supplementation in particular since its washout period is approximately four weeks (Hultman et al., 1996; Febbraio et al., 1995). Subjects were instructed to avoid any form of exercise 24 hours before testing and any exercise involving intense lower leg exercises for at least 48 hours before testing. Recruitment was through advertisement and a detailed information sheet was provided to all participants before they signed an informed consent. The study was approved by the Ethics Committee of the Carnegie Faculty at Leeds Beckett University whilst all participants completed medical and training history questionnaires prior to any testing.

\section{Outline of Experimental Procedures}

Following screening and baseline testing, participants were randomly assigned to one of four experimental groups [Placebo, (PLA, n=7), Creatine $(\mathrm{Cr}, \mathrm{n}=8)$, Sodium Bicarbonate $(\mathrm{SB}, \mathrm{n}=6)$, and a combined $\mathrm{Cr}+\mathrm{SB}(\mathrm{n}=6)]$. They then followed a standardised diet plan for a period of four days whilst taking a specified daily amount of one of the supplements above before they completed an exercise testing trial on day five. No supplements were taken on the day of testing. An overview of the adopted tests and testing procedures is provided in Figure 1. 
Figure 1. Outline of Testing Procedures. One Repetition Maximum Strength (1RM)

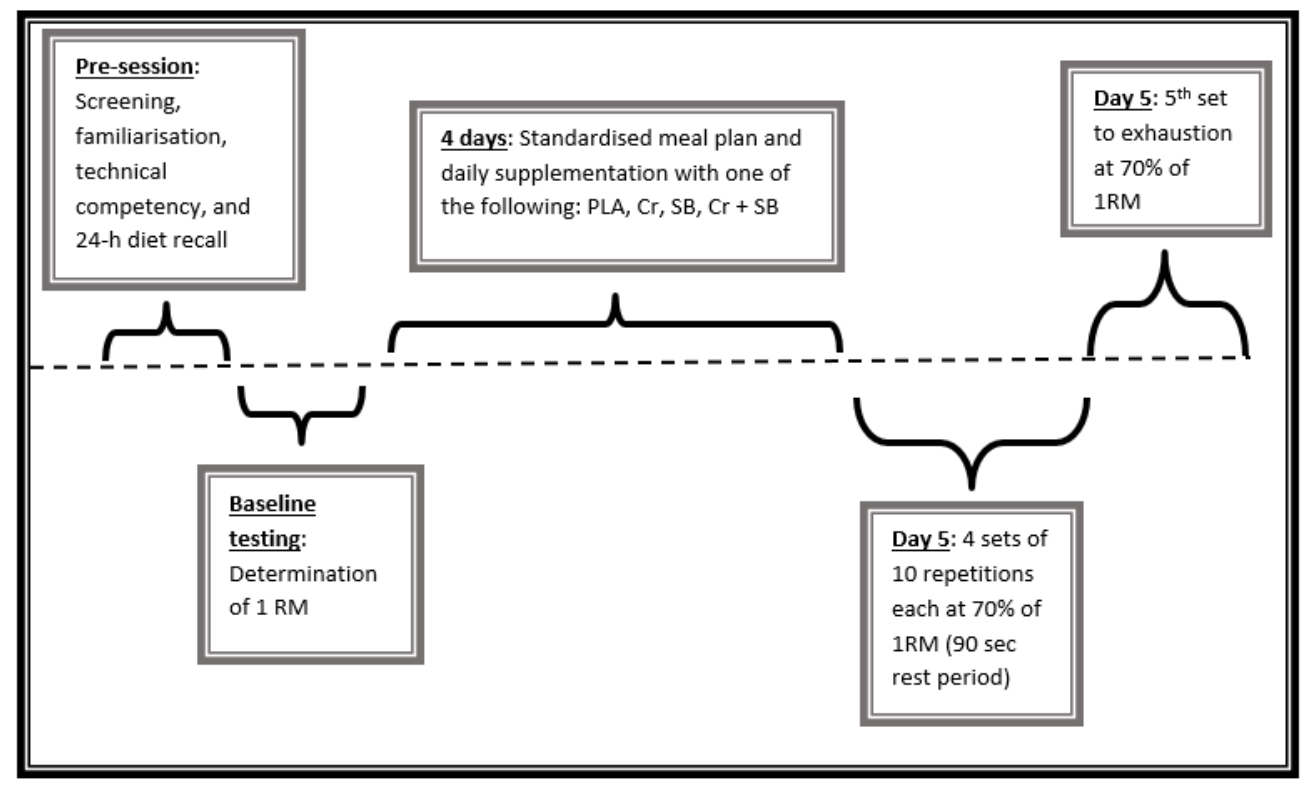

\section{One Repetition Maximum (1RM) Strength Testing and 24 h Dietary Recall}

Baseline one repetition maximum (1RM) strength testing took place following screening and assessment of the technical competency in the back squat exercise by an accredited coach. Prior to 1RM testing, the participants also completed a $24 \mathrm{~h}$ food recall under the supervision of a dietician along with a list of common foods, so as to analyse their eating habits and base their diet plan on their food preferences. Participants who were deemed to be technically competent had their stature (Seca, Alpha 770, Germany), body mass (Seca, Alpha 888, Germany), blood lactate (BL) and blood glucose (BG) concentration (YSI, 2300 Stat Plus, Ohio, USA) measured. The back squat was chosen as an exercise which utilises large muscle groups, it comprises a key feature of typical strength and conditioning sessions, and it can result in high levels of acidosis (Webster et al., 1993; Nicholson et al., 2014). Participants completed the 1RM tests in the same laboratory using a customised power rack with adjusted safety stoppers and at the same time of day after four hours of fasting.

Following five minutes standardised warm-up on a cycle ergometer participants had their 1RM measured using procedures identical to those described previously (Baechle et al., 2000). Participants' 1RM was measured within three to five attempts and approximately four to five minutes of rest was permitted between attempts. Successful attempts required participants to descend to the point where the tops of the thighs were parallel to the floor and squat depth was visually assessed by the same qualified trainer. Shoulder girth was also measured and multiplied by 1.5 to standardise the width of stance which the participants would use during squatting. Tape was used to mark the desired foot positioning, and the inside of the heels positioned to the outside of 
the tape. Participants were instructed to squat so that their knee joint flexion decreased to $90^{\circ}$ at the bottom of the lift. The qualified trainer was positioned to the side of the participant and called 'parallel' once the participant's knee was at $90^{\circ}$ flexion. A spotter was provided for every back squat completed to ensure safety whilst lifting.

\section{Diet and Supplementation}

Based on the findings of the $24 \mathrm{~h}$ dietary recall a qualified dietician prepared the 4-day dietary plan which was composed of 55-60\% carbohydrate, $20-25 \%$ fat and $15-20 \%$ protein. The macronutrient contributions to total energy intake were estimated based on recommendations by the American Dietetic Association, Dieticians of Canada, and the American College of Sports Medicine (Rodriguez et al., 2009) as well as the typical eating practices of the participants. Total energy requirements (TER) were calculated using the Harris-Benedict equation to estimate basal metabolic rate (BMR; Roza and Shizgal, 1984) and a physical activity factor (Alfonzo-González et al., 2004). The standardised meal was planned according to the participants' energy requirements, in an attempt to ensure that none of the participants consumed below or above their daily estimated energy and macronutrient requirements. In addition to the standardised meal plan, participants also ingested on a daily basis one of the following: 1) PLA (corn flour); 2) $\mathrm{Cr}$ (creatine monohydrate, MYPROTEIN); 3) SB (Bell's, Sodium Bicarbonate BP); 4) $\mathrm{Cr}+\mathrm{SB}$. The supplements were packed into gelatine capsules and each participant received a number of capsules relative to their body mass. All capsules were prepared under antiseptic conditions in a nutrition laboratory. Since the combined condition would result in the highest number of capsules for any participant, placebo capsules containing corn flour were used to increase the total number of capsules given to the PLA, SB and $\mathrm{Cr}$ groups to match the number that would be given to the combined group. The daily dosages were: $\mathrm{Cr}=20 \mathrm{~g}^{-1}$ plus PLA; $\mathrm{SB}=0.5 \mathrm{~g} \mathrm{~kg}^{-1} \cdot \mathrm{d}^{-1}$ of SB plus PLA; $\mathrm{Cr}+\mathrm{SB}=5 \mathrm{~g}^{-1}$ of $\mathrm{Cr}$ plus $0.5 \mathrm{~g} \cdot \mathrm{kg}^{-}$ ${ }^{1} \cdot \mathrm{d}^{-1} \mathrm{SB}$; PLA = same number of capsules as the $\mathrm{Cr}+\mathrm{SB}$. The daily amount was divided in four equal doses and placed in separate bags whilst participants were instructed to take the first dose in the morning and the remaining ones every three to four hours thereafter.

The supplementation regime involved serial loading for four days since this is associated with reduced gastrointestinal (GI) discomfort following SB supplementation (Sahlin, 2014; Burke and Pyne, 2007). This duration is also considered adequate for positive changes in relation to either SB supplementation (Sahlin, 2014; McNaughton et al., 2008; McNaughton et al., 1999) or Cr (Buford et al., 2007; Sahlin, 2014; Hultman et al., 1996). Sickness and stomach ache levels (Van Montfoort et al., 2004) were recorded every time participants took the supplements. Participants were also advised to take the supplements alongside food since this may enhance absorption rates of $\mathrm{Cr}$ (Buford et al., 2007; Steenge et al., 2000; Green et al., 1996), and to drink water up to $500 \mathrm{ml}$ in an attempt to minimise levels of GI discomfort every 
time they took the supplements. In order to ensure that participants adhered to the supplementation requirements, text messages were sent to them as reminders every time they had to take the required dose. For control purposes, a standardised meal plan was prescribed to all participants. Higher protein intakes are associated with higher $\mathrm{Cr}$ concentration (Sahlin, 2014) whilst higher carbohydrate intakes with increased muscle glycogen levels (Bergström et al., 1967) which is one of the major sources of energy during hypertrophy type training (Lambert and Flynn, 2002). Therefore, it was crucial to control the dietary intake of participants in an attempt to make meaningful conclusions in relation to the ergogenic potential of the supplements under investigation.

\section{Resistance Exercise Bout (REB)}

On day five, following four days supplementation with one the supplements outlined above, participants completed a REB (4x10 repetitions at $70 \%$ of $1 \mathrm{RM}$ with $1 \frac{1}{2}$ min recovery between sets), they then had another $1 \frac{1}{2}$ min rest after the fourth set before they completed a fifth set at $70 \%$ of $1 \mathrm{RM}$ to exhaustion. Before the REB the same standardised warm-up as in 1RM testing was employed. Ratings of perceived exertion using the Borg Scale (Borg, 1982) were measured at the end of each set. The duration of each set was recorded during each set using a stopwatch to allow calculation of the total time-under-tension. Heart rate (HR) was also recorded during each set using a radio telemetry monitor (Polar Vantage NV, Kempele, Finland). In cases where participants failed to complete the expected number of repetitions or stood still for over five seconds, the weight was reduced in subsequent sets to allow for successful completion of the required repetitions. Fingertip blood samples were drawn before the warm up, and immediately after completion of the fourth and fifth sets to measure BG and BL concentration. Approximately $25 \mu \mathrm{L}$ of blood was drawn using a disposable lancet (Kendall, MonoletMonoject, UK) and collected in a heparin-lined capillary tube (Sarstedt, Microvette CB 300, Germany). The samples were analysed in duplicate using a calibrated YSI 2300 STAT Plus analyser (Yellow Springs, USA).

\section{Statistical Analysis}

All data were analysed using SPSS (version 20.0, Chicago, IL). In order to ensure normality, data were tested for normal distribution using the ShapiroWilk test because the number of subjects was less than 50. Homogeneity of variance was tested using Levene's test. Adjustment was made using the Brown-Forsythe test when homogeneity was violated. A one-way repeated measures analysis of variance (ANOVA) was employed to examine the effect of supplementation on blood related variables, duration of four sets, heart rate and ratings of perceived exertion. Homogeneity of variance was tested by Mauchly's test of Sphericity. When significant main effects were observed, Fisher's Least Significant Difference (LSD) test with Gabriel adjustment were employed to determine where the differences lied. Non-parametric tests 
(Kruskall Wallis) were employed when there was violation of the assumptions for use of parametric tests. Comparisons for number of repetitions completed in relation to the placebo were also performed using Cohen's Effect Size (ES) with threshold values for small (0.2), medium (0.6), large (1.2), very large (2.0) and extremely large (4.0). All data are presented as mean $\pm \mathrm{SD}$, statistical significance was set at $\mathrm{p}<0.05$.

\section{Results}

Mean anthropometric characteristics and 1RM for participants in all groups are given in Table 1. Twenty seven participants completed all tests and testing procedures (PLA, $\mathrm{n}=7 ; \mathrm{Cr}, \mathrm{n}=8 ; \mathrm{SB}, \mathrm{n}=6$; and $\mathrm{Cr}+\mathrm{SB}, \mathrm{n}=6$ ).

Table 1. Mean ( $\pm S D)$ Age, Height, Body Mas, and 1RM of Participants in each Group. Abbreviations: $P L A=$ Placebo, $C r=$ Creatine, $S B=$ Sodium Bicarbonate, IRM= One Repetition Maximum Strength

\begin{tabular}{|l|c|c|c|c|}
\hline Variables & $P L A(n=7)$ & $C r(n=8)$ & $S B(n=6)$ & $\begin{array}{c}C r+S B \\
(n=6)\end{array}$ \\
\hline Age (years) & $31.4( \pm 7.7)$ & $24.00( \pm 1.9)$ & $24.7( \pm 2.9)$ & $27.3( \pm 5.8)$ \\
\hline Body Mass $(\mathrm{kg})$ & $77.0( \pm 4.6)$ & $78.1( \pm 8.9)$ & $76.0( \pm 7.3)$ & $79.2( \pm 8.5)$ \\
\hline Height $(\mathrm{cm})$ & $\begin{array}{c}174.1 \\
( \pm 7.2)\end{array}$ & $176.6( \pm 5.4)$ & $177.8( \pm 6.9)$ & $175.4( \pm 6.9)$ \\
\hline $1 R M(\mathrm{~kg})$ & $120( \pm 22.0)$ & $\begin{array}{c}103.1 \\
( \pm 12.3)\end{array}$ & $124.2( \pm 36.0)$ & $\begin{array}{c}142.5 \\
( \pm 31.3)\end{array}$ \\
\hline
\end{tabular}

Creatine supplementation resulted in significant gains in body mass following 4 days of supplementation (from $78.1 \pm 8.9 \mathrm{~kg}$ pre-supplementation to $79.1 \pm$ $8.9 \mathrm{~kg}$ post-supplementation, $\mathrm{p}<0.05$ ) (Table 2 ).

Table 2. Mean Difference $( \pm S D)$ in Body Mass Change from Pre- to Postsupplementation Period. Abbreviations: PLA=Placebo, Cr=Creatine, $S B=$ Sodium Bicarbonate

\begin{tabular}{|l|c|c|c|c|}
\hline Variables & $P L A(n=7)$ & $C r(n=8)$ & $S B(n=6)$ & $\begin{array}{c}C r+S B \\
(n=6)\end{array}$ \\
\hline Minimum $(\mathrm{kg})$ & -1.2 & -1.0 & -1.0 & -1.0 \\
\hline Maximum $(\mathrm{kg})$ & 1.2 & 2.2 & 1.1 & 1.2 \\
\hline Mean difference $(\mathrm{kg})$ & $-0.3( \pm 0.8)$ & $1.1( \pm 1.0)$ & $-0.02( \pm 0.8)$ & $0.5( \pm 0.9)$ \\
\hline
\end{tabular}

\section{Performance Outcomes}

During the fifth set participants managed to complete successfully 8 $( \pm 1.6), 11( \pm 5.5), 9( \pm 3.7)$, and $11( \pm 3.3)$ repetitions in the PLA, Cr, SB and $\mathrm{Cr}+\mathrm{SB}$ groups, respectively. Despite the fact that no significant differences were observed overall $(\mathrm{p}=0.379)$ in relation to the total number of repetitions performed during the fifth set, small and moderate effect sizes (ES) were observed between the PLA and the remaining experimental groups. In 
particular, a small ES (0.4) was observed in the SB group whilst moderate ES were observed for both the creatine (0.7) and combined (1.0) groups. Participants in the PLA group completed the required number of repetitions at slightly lower intensity of 1RM $(68 \pm 3.4 \%)$ than required however all the remaining groups completed the target repetitions at the target intensity.

The mean time to complete the four sets was $121.2( \pm 14.3), 120.0( \pm$ 13.3), $116.2( \pm 17.2), 116.4$ ( \pm 16.4$)$ seconds for the PLA, Cr, SB, and $\mathrm{Cr}+\mathrm{SB}$ groups, respectively (Figure 2 ). The statistical analysis showed a significant time effect in the duration of sets $(\mathrm{p}=0.033)$ and post-hoc analysis showed that set four was significantly longer than set three in the SB group $(\mathrm{p}=0.039)$. Overall, no significant differences were observed between groups in relation to the time it took participants to complete each set $(\mathrm{p}=0.632)$. Despite the fact that no significant differences existed between groups the $\mathrm{SB}$ and $\mathrm{Cr}+\mathrm{SB}$ groups completed the four sets on average faster than the remaining groups. Moreover, small ES were observed between SB (-0.3) and $\mathrm{Cr}+\mathrm{SB}(-0.3)$ in relation to the PLA group.

Figure 2. Mean Time in Seconds to Complete each Set During the Experimental Protocol. Error Bars Denote Standard Deviations. Abbreviations: PLA=Placebo, $C r=$ Creatine, $S B=$ Sodium Bicarbonate

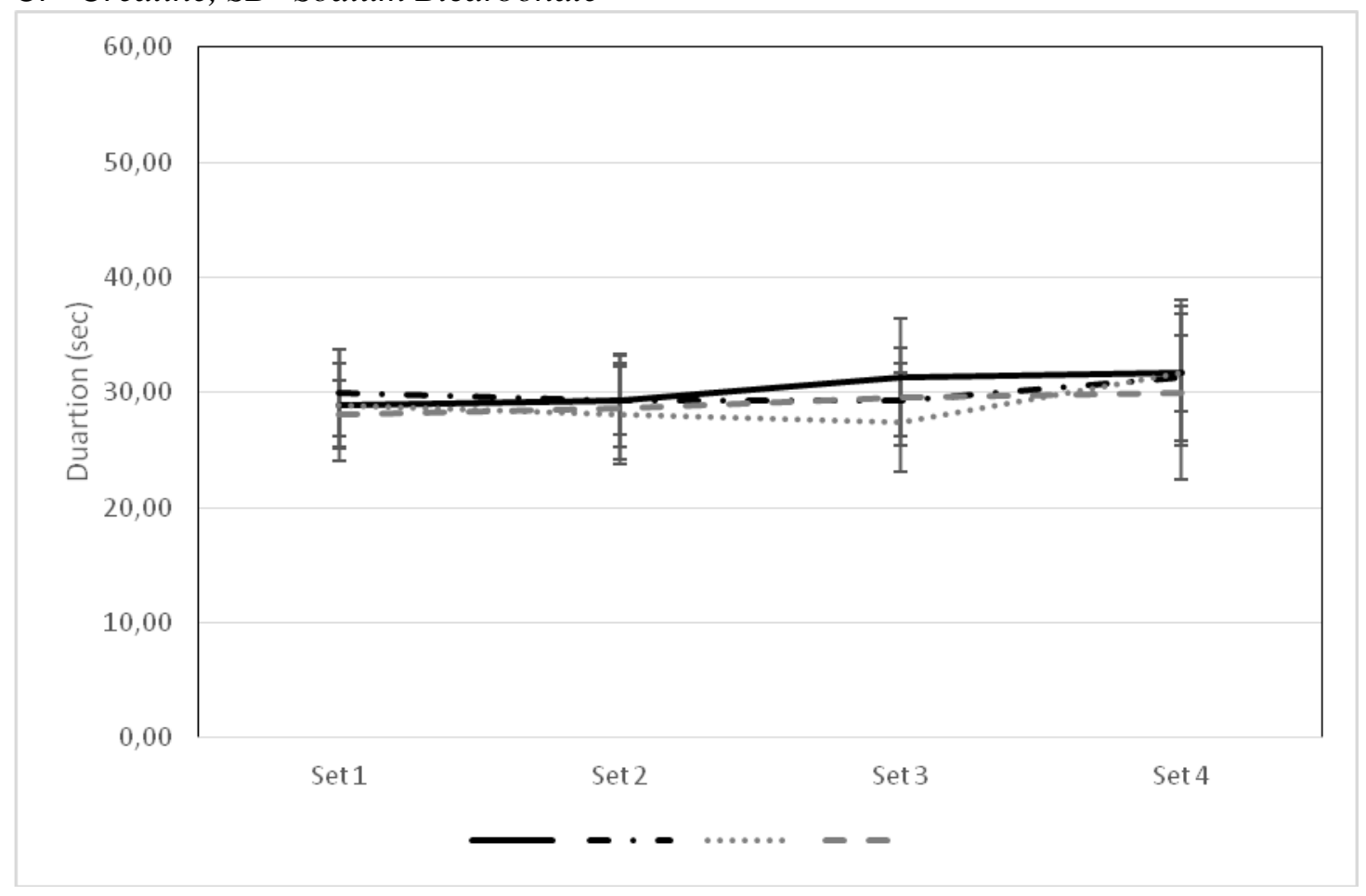

Ratings of Perceived Exertion and Heart Rate

Ratings of perceived exertion (RPE) during the four sets were on average $15.5( \pm 1.7), 15.4( \pm 1.7), 16.0( \pm 0.9)$, and $16.2( \pm 0.9)$ for the PLA, Cr, SB, and $\mathrm{CR}+\mathrm{SB}$ groups, respectively. Significant differences in RPE were not observed during the four training sets however a significant main group effect $(\mathrm{p}=0.002)$ 
was observed when the fifth set was taken into account (Table 3). Post hoc analysis showed that during the fifth set RPE in the PLA group was significantly lower than $\mathrm{Cr}(\mathrm{p}=0.014), \mathrm{SB}(\mathrm{p}=0.025)$ and $\mathrm{Cr}+\mathrm{SB}(\mathrm{p}=0.001)$.

Table 3. Mean Ratings of Perceived Exertion $( \pm S D)$ during Exercise. Abbreviations: $P L A=$ Placebo, $C r=$ Creatine, $S B=$ Sodium Bicarbonate

\begin{tabular}{|c|c|c|c|c|}
\hline Sets & $P L A(n=7)$ & $C r(n=8)$ & $S B(n=6)$ & $\begin{array}{c}C r+S B \\
(n=6)\end{array}$ \\
\hline 1 & $14.7( \pm 1.7)$ & $\begin{array}{c}13.8( \pm \\
1.6)\end{array}$ & $13.7( \pm 1.0)$ & $14.3( \pm 1.2)$ \\
\hline 2 & $15.6( \pm 2.3)$ & $\begin{array}{c}14.6( \pm \\
1.5)\end{array}$ & $15.8( \pm 1.0)$ & $16.0( \pm 0.9)$ \\
\hline 3 & $15.7( \pm 1.8)$ & $\begin{array}{c}15.9( \pm \\
2.0)\end{array}$ & $16.8( \pm 1.0)$ & $16.7( \pm 1.2)$ \\
\hline 4 & $16.0( \pm 1.4)$ & $\begin{array}{c}17.3( \pm \\
2.6)\end{array}$ & $17.8( \pm 1.2)$ & $17.7( \pm 0.8)$ \\
\hline 5 & $16.7( \pm 1.8)$ & $\begin{array}{c}19.0( \pm \\
1.3)\end{array}$ & $19.0( \pm 1.1)$ & $19.8( \pm 0.5)$ \\
\hline
\end{tabular}

Heart rate was similar in all groups with no significant differences observed between groups at any stage (Figure 3 ).

Figure 3. Mean Heart Rate during the Exercise Testing Protocol. Error Bars Denote Standard Deviations. Abbreviations: PLA=Placebo, Cr=Creatine, $S B=$ Sodium Bicarbonate

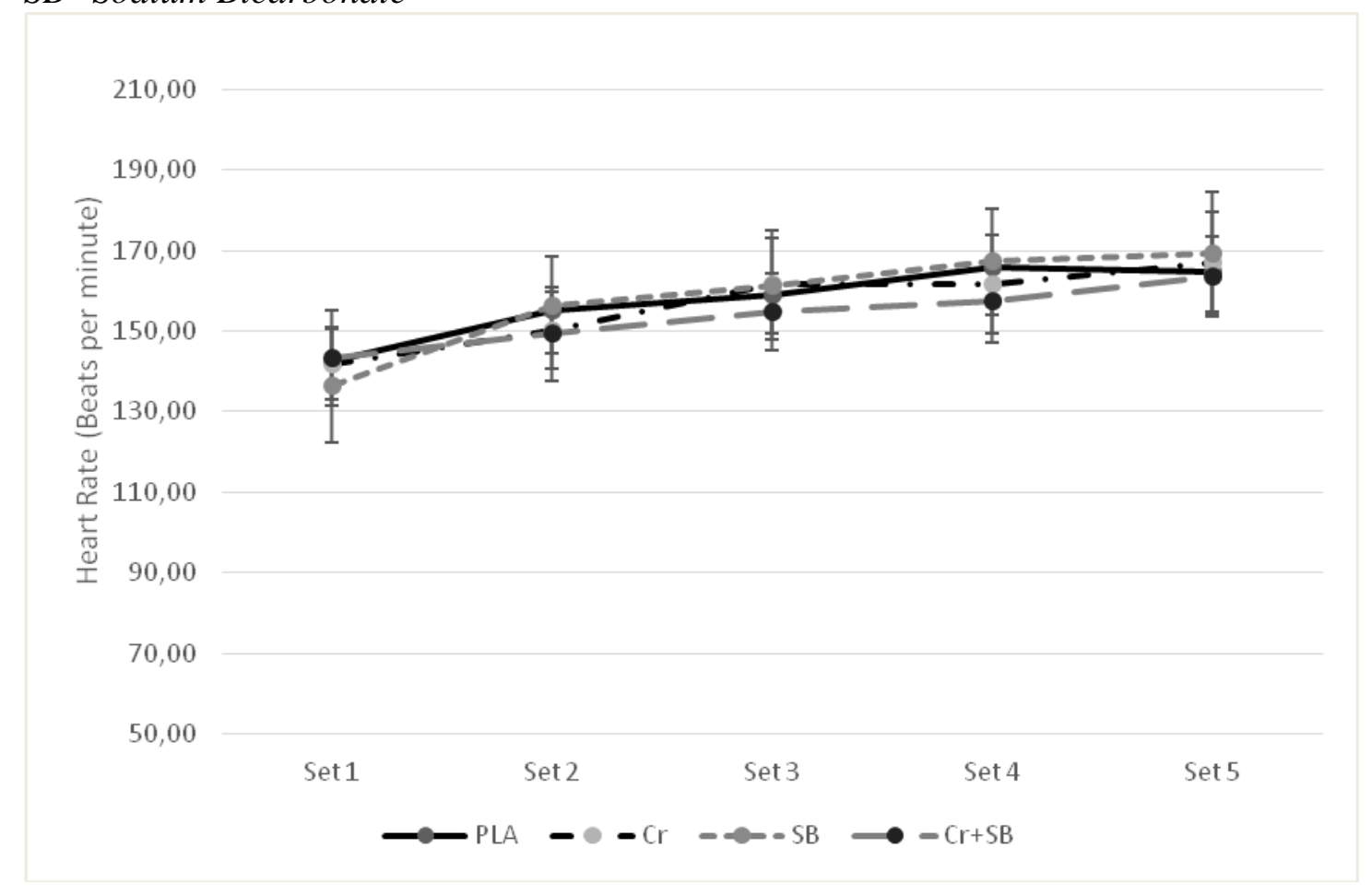




\section{Blood Chemistry Variables}

Average blood glucose concentration including the pre- and post-exercise values was $4.6( \pm 0.6), 4.5( \pm 0.3), 4.6( \pm 0.2)$, and $4.3( \pm 0.4) \mathrm{mmol}^{-1}$ for the PLA, Cr, SB, and CR+SB groups respectively. Significant differences were not observed between or within groups at any time point during the exercise testing trial (Figure 4).

Figure 4. Blood Glucose Concentration $\left(\mathrm{mmol} \mathrm{L}^{-1}\right)$ during Exercise Testing. Error Bars Denote Standard Deviations. Abbreviations: PLA=Placebo, $C r=$ Creatine, $S B=$ Sodium Bicarbonate

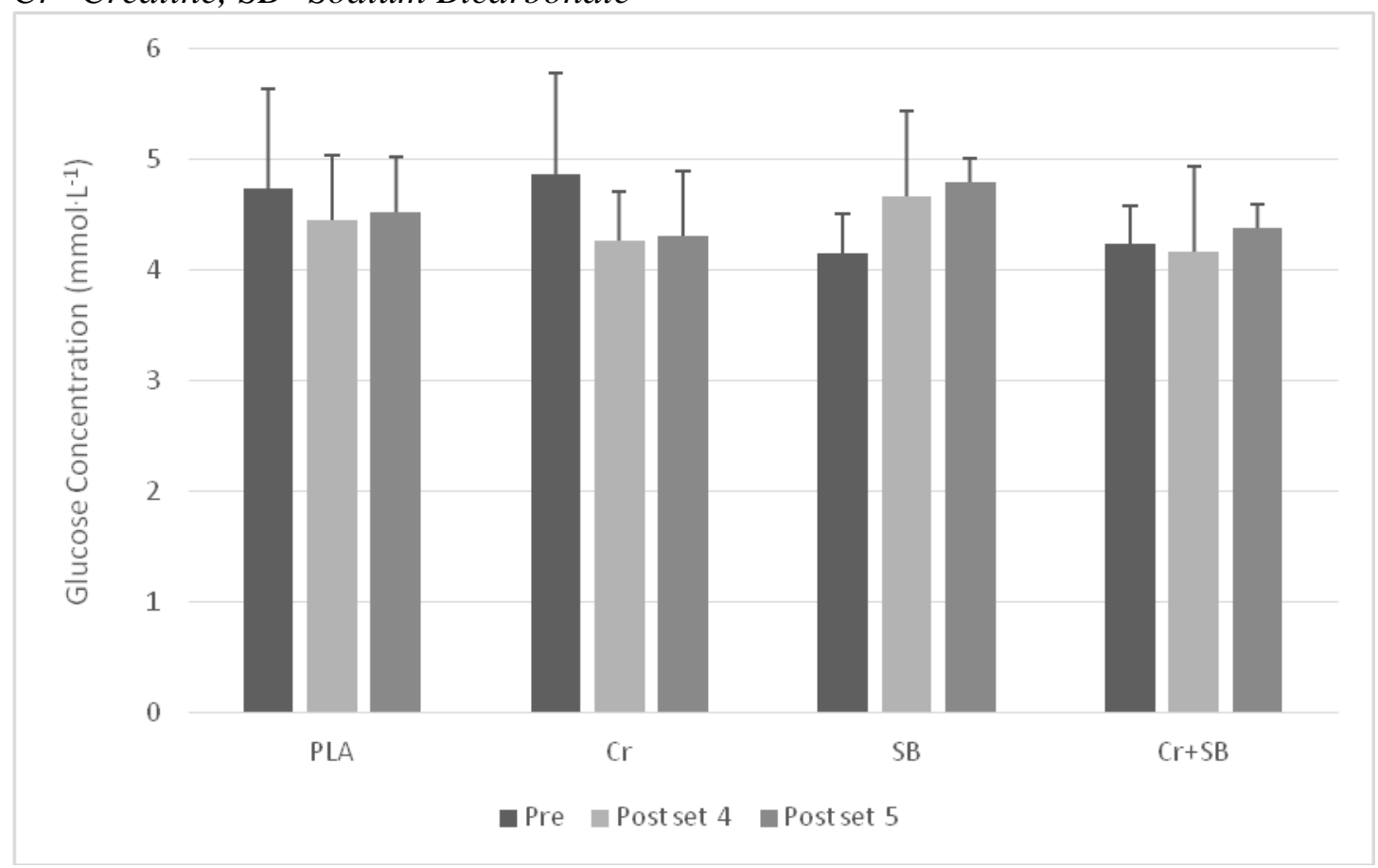

Average blood lactate concentration including the pre- and post-exercise values was $4.9( \pm 1.0), 4.3( \pm 1.0), 5.6( \pm 1.5)$, and $4.8( \pm 0.5) \mathrm{mmol}^{-1}$ for the PLA, Cr, SB, and CR+SB groups respectively. Significant differences were not observed between groups at any time point during the exercise testing trial however significant time effects were observed $(\mathrm{p}=0.00001)$ where postexercise blood lactate concentration in all groups were significantly higher than the corresponding blood lactate concentration pre-exercise (Figure 5). 
Figure 5. Blood Lactate Concentration ( $\mathrm{mmol}^{-1}$ ) during Exercise Testing. Error Bars Denote Standard Deviations. Abbreviations: PLA=Placebo, $C r=$ Creatine, $S B=$ Sodium Bicarbonate

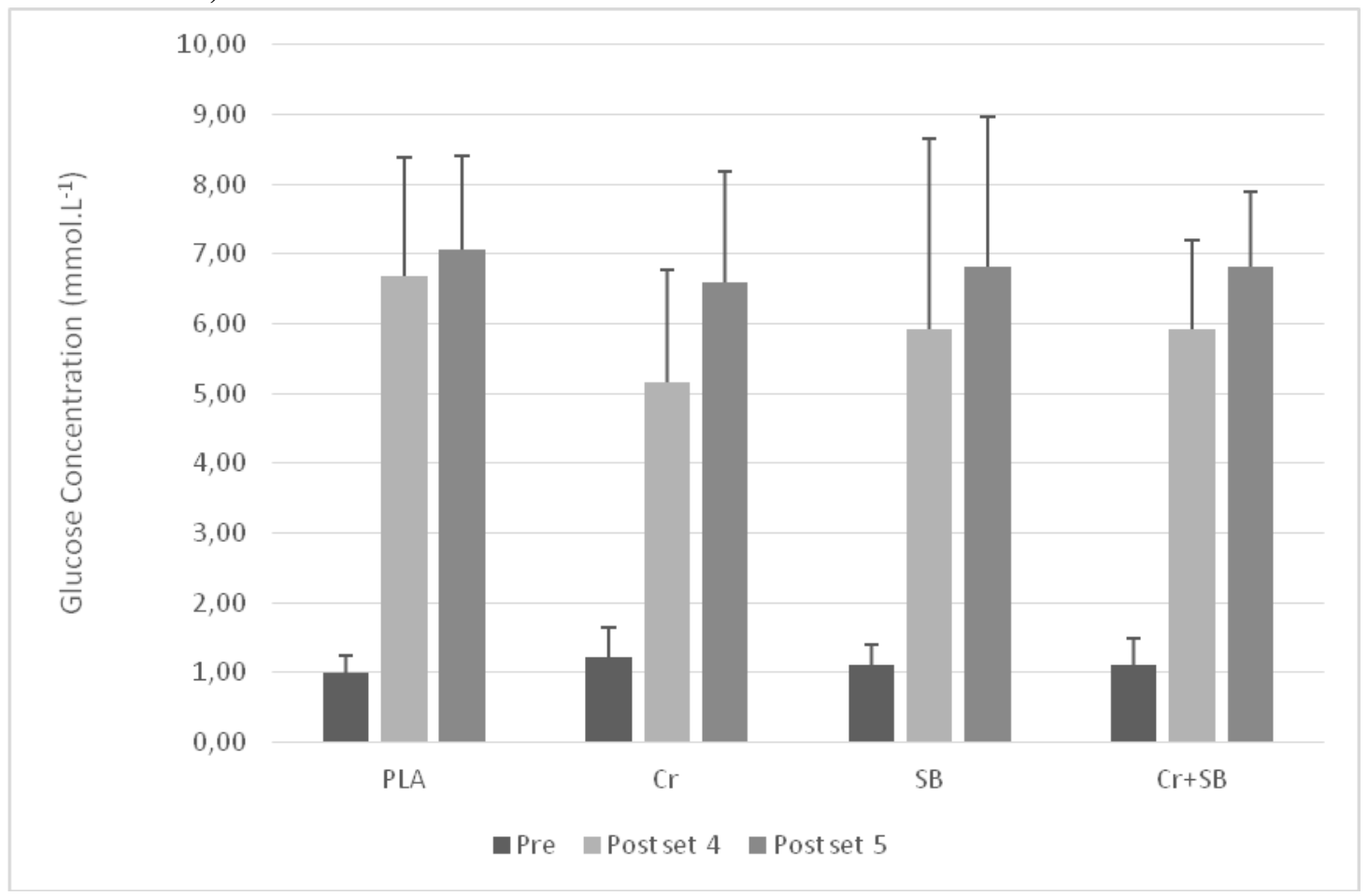

\section{Discussion}

The primary purpose of this study was to examine whether combined supplementation of creatine $(\mathrm{Cr})$ with sodium bicarbonate $(\mathrm{SB})$ would further enhance performance and reduce fatigue rates, compared to placebo (PLA), Cr, and SB alone. Our findings did not confirm the hypothesis that $\mathrm{Cr}+\mathrm{SB}$ would further enhance performance in the back squat exercise than $\mathrm{Cr}$ alone. However, participants in the $\mathrm{Cr}+\mathrm{SB}$ completed the same number of repetitions during the fifth set (main performance outcome) as participants in the Cr group suggesting that when smaller amounts of $\mathrm{Cr}$ are taken with $\mathrm{SB}$ the same ergogenic effect may be achieved as when larger amounts of $\mathrm{Cr}$ are ingested. In relation to the number of repetitions completed during the fifth set significant differences did not exist between groups however the moderate effects sizes (ES) observed between $\mathrm{Cr}$, and $\mathrm{Cr}+\mathrm{SB}$ in relation to PLA provide support for the effectiveness of $\mathrm{Cr}$, and $\mathrm{Cr}+\mathrm{SB}$ as ergogenic aids during hypertrophy type training programmes. SB ingestion was also associated with performance benefits in relation to the PLA (ES: 0.4) however they were of smaller magnitude compared to the creatine containing groups. Taking also into account that in the PLA group, adjustments had to be made in the weight lifted during the four sets $(68 \pm 3.4 \%$ of $1 \mathrm{RM}$ instead of $70 \%$ of $1 \mathrm{RM})$, it is highly probable that the magnitude of performance gains in the three experimental groups $(\mathrm{Cr}, \mathrm{SB}$, and $\mathrm{Cr}+\mathrm{SB})$ would have been greater in relation to the PLA 
group. The hypothesis that $\mathrm{Cr}$ supplementation causes water retention (Greenhaff et al., 1994; Sahlin, 2014) was confirmed in our study. Participants in the Cr group had significant increases in their body mass however under the current circumstances this increase was not associated with a negative impact on performance. It is likely that the body mass gains would have negatively affected performance in sports where an increase in the power-to-body mass ratio is essential for success however we did not test this hypothesis.

Our findings are in agreement with research findings that have supported the ergogenic potential of combining $\mathrm{Cr}+\mathrm{SB}$ when compared to a placebo (Barber et al., 2013; Mero et al., 2004; Griffen et al., 2015). The greatest attenuation in the decline of peak power in participants in the study by Barber et al. (2013) suggests that $\mathrm{Cr}+\mathrm{SB}$ may be more beneficial than $\mathrm{Cr}$ alone however others do not support additive performance benefits of $\mathrm{Cr}+\mathrm{SB}$ when compared to creatine (Griffen et al., 2015). In our study, small ES (-0.3) were observed between SB and PLA, and $\mathrm{Cr}+\mathrm{SB}$ and PLA in relation to the time it took participants to complete the four sets. This trend provides some support for a synergistic benefit since the addition of SB seems to allow participants to complete the sets in a shorter time. The discrepancy between our study and the study by Griffen at al. (2015) may be due to methodological differences and more specifically the SB dosage $\left(0.3 \mathrm{~g} \mathrm{~kg}^{-1} \cdot \mathrm{d}^{-1}\right.$ vs $\left.0.5 \mathrm{gkg}^{-1} \cdot \mathrm{d}^{-1}\right)$ and duration of supplementation (seven versus four days). Taking also into account the short duration of the previous study it is likely that there was a carry-over effect due to inadequate washout period between conditions. In our study, co-ingestion of small $\mathrm{Cr}$ amount $\left(5 \mathrm{~g} \mathrm{~d}^{-1}\right)$ with an amount of $\mathrm{SB}\left(0.5 \mathrm{~g} \mathrm{~kg}^{-1} \cdot \mathrm{d}^{-1}\right)$ that is considered optimum (Burke and Pyne, 2007; McNaughton et al., 1999) for serial loading, seem to be equally effective as a standard $\mathrm{Cr}$ dose $\left(20 \mathrm{~g}^{-1} \mathrm{~d}^{-1}\right)$ regarding the attenuation of fatigue since participants in the $\mathrm{Cr}$ and $\mathrm{Cr}+\mathrm{SB}$ groups performed the required number of repetitions faster than the remaining groups. This may be a promising trend however more studies are required to confirm the findings due to the small observed ES and sample size in our study.

In relation to performance outcomes, there was a clearer trend for ergogenic benefits during the fifth set regardless of the buffer or combination of buffers (Cr, SB, and $\mathrm{Cr}+\mathrm{SB})$ used. On this performance aspect, we did observe similar ES as others (Griffen et al., 2015) with moderate ES (0.7-1.0) between $\mathrm{Cr}, \mathrm{Cr}+\mathrm{SB}$ and PLA, and small ES (0.4) between SB and PLA, which suggests that $\mathrm{Cr}, \mathrm{SB}$, and $\mathrm{Cr}+\mathrm{SB}$ may enhance performance to greater extent than a placebo. Moreover, the largest ES (1.0) observed in the combined group also suggests that the magnitude of performance gains may be greater when combining these two buffers. Another of the advantages of the current mode of supplementation was that serial loading was not associated with any gastrointestinal discomfort providing further support for this mode of ingestion as a means to enhance performance.

Ratings of perceived exertion (RPE) were similar between all groups during the four sets however significantly lower in the PLA group compared to the remaining experimental groups during the fifth set. The explanation for this 
difference may be due to the fact that participants in the PLA group performed fewer repetitions at a slightly lower intensity (68\% of 1RM instead of $70 \%$ of $1 \mathrm{RM}$ ) during the fifth set since adjustments had to be made in the fourth set to enable participants to perform the required number of sets and repetitions. Moreover, a larger standard deviation in the PLA group indicates a larger variation in the RPE scores reached by participants in the PLA group during the fifth set. Even though participants were expected to be of similar training status, the baseline strength data suggests that some of them may have been more trained than others. Therefore it is probable that training status varied between individuals. It is known that more trained participants are more likely to have enhanced buffering capacity as result of training (Sharp et al., 1986; Parkhouse and McKenzie, 1984; Sahlin, 2014) however in our study the weakest group $(\mathrm{Cr})$ and the strongest group $\mathrm{Cr}+\mathrm{SB}$ had similar responses therefore it is unlikely that this was the case. A number of inclusion and exclusion criteria as well as the fact that during the exercise testing protocol the intensity that was used was relative to the participants' 1RM are key control mechanisms that were in place as means to overcome such limitations. Additionally, the potential enhanced buffering capacity resulting from supplementation with $\mathrm{Cr}$ and SB more likely enabled participants to work harder during the last set, and this was reflected in perceived exertion ratings. Overall, the heart rate and blood lactate data suggests that participants in all groups physiologically pushed themselves to similar extent during the four sets and reached similar levels of volitional exhaustion during the fifth set with the only exception in RPE during the fifth set.

In our study, participants were randomly assigned to one of four discreet groups. With the current sample size, a crossover design would have enabled us to make more concrete conclusions in relation to the ergogenic potential of combining $\mathrm{Cr}+\mathrm{SB}$ since participants would have acted as their own controls. However, a crossover design with four conditions would have created a number of other challenges due to the long washout period for creatine which is approximately four weeks (Hultman et al., 1996, Febbraio et al., 1995). Therefore the current experimental approach was the most appropriate under the circumstances. A larger sample size would have overcome some of the limitations associated with experimental research involving discreet groups however the main objective of this pilot study was to explore trends. Particular emphasis was placed on putting control mechanisms in place that would minimise the impact of confounding variables such as training status or diet. The impact of training status was discussed in previous paragraphs. Strict dietary controls and carefully chosen inclusion/exclusion criteria were put in place in an attempt to reduce the chances of heterogeneity between groups. For example, participants with low initial creatine stores such as vegans or vegetarians (Maccormick et al., 2004; Burke et al., 2003; Shomrat et al., 2000) are more likely to respond positively when supplemented with creatine (responders) compared to individuals with high baseline creatine stores (nonresponders) (Cooper et al., 2012). Energy and macronutrient intakes were also carefully monitored and controlled since an increase in the carbohydrate intake 
in particular would have enhanced muscle creatine uptake (Steenge et al., 1998). In our study, all participants received the same relative macronutrient intake and daily energy intake according to recommendations (Rodriguez et al., 2009; Roza and Shizgal, 1984; Alfonzo-González et al., 2004) and this was also supported by our blood glucose data which was similar in all groups.

In conclusion, our study provides additional support for the ergogenic potential of $\mathrm{Cr}+\mathrm{SB}$ during hypertrophy type resistance training since performance benefits were of similar magnitude to a standard $\mathrm{Cr}$ group, whilst the addition of SB seems to be associated with completion of the repetitions in a shorter time. One of the advantages of our current dosage protocol is that smaller amount of $\mathrm{Cr}\left(5 \mathrm{~g} \mathrm{~d}^{-1}\right)$ co-ingested with $\mathrm{SB}\left(0.5 \mathrm{~g} \mathrm{~kg}^{-1} \cdot \mathrm{d}^{-1}\right)$ could be equally effective as larger amount of $\mathrm{Cr}\left(20 \mathrm{gd}^{-1}\right)$ without a concomitant increase in body mass. This can be particularly beneficial in sports where and increase in the power-to-body mass ratio is essential for successful performance. In addition, our study also confirmed that serial ingestion of relatively large amounts of SB does not result in gastrointestinal discomfort therefore can be part of the ergogenic aids portfolio of athletes in high intensity sports. Further research with larger sample size is necessary to confirm the findings of the current study.

\section{References}

Ahtiainen, J. P., Pakarinen, A., Alen, M., Kraemer, W. J. \& Hakkinen, K. (2003). Muscle hypertrophy, hormonal adaptations and strength development during strength training in strength-trained and untrained men. Eur J Appl Physiol, 89, $555-63$.

Alfonzo-González, G., Doucet, E., Alméras, N., Bouchard, C. \& Tremblay, A. (2004). Estimation of daily energy needs with the FAO/WHO/UNU 1985 procedures in adults: comparison to whole-body indirect calorimetry measurements. European journal of clinical nutrition, 58, 1125-1131.

Andersen, J. L. \& Aagaard, P. (2010). Effects of strength training on muscle fiber types and size; consequences for athletes training for high-intensity sport. Scand $J$ Med Sci Sports, 20 Suppl 2, 32-8.

Baechle, T. R. \& Earle, R. W. (2008). Essentials of strength training and conditioning, Leeds, Human Kinetics.

Baechle, T. R., Earle, R. W. \& Wathen, D. (2000). Resistance training. In: BAECHLE, T. R. \& EARLE, R. W. (eds.) Essentials of strength training and conditioning. 2 ed.: Human Kinetics.

Barber, J. J., Mcdermott, A. Y., Mcgaughey, K. J., Olmstead, J. D. \& Hagobian, T. A. (2013). Effects of combined creatine and sodium bicarbonate supplementation on repeated sprint performance in trained men. J Strength Cond Res, 27, 252-8.

Bergström, J., Hermansen, L., Hultman, E. \& Saltin, B. (1967). Diet, muscle glycogen and physical performance. Acta Physiologica Scandinavica, 71, 140-150.

Borg, G. A. V. (1982). Psychophysical bases of perceived exertion. / Les bases psychophysiques de la perception de l' effort. Medicine \& Science in Sports \& Exercise, 14, 377-381.

Buford, T. W., Kreider, R. B., Stout, J. R., Greenwood, M., Campbell, B., Spano, M., Ziegenfuss, T., Lopez, H., Landis, J. \& Antonio, J. (2007). International Society 
of Sports Nutrition position stand: creatine supplementation and exercise. Journal of the International Society of Sports Nutrition, 4, 1-8.

Burke, D. G., Chilibeck, P. D., Parise, G., Candow, D. G., Mahoney, D. \& Tarnopolsky, M. (2003). Effect of creatine and weight training on muscle creatine and performance in vegetarians. Med Sci Sports Exerc, 35, 1946-55.

Burke, L. (2007). Practical sports nutrition, Leeds, Human Kinetics.

Burke, L. \& Pyne, D. (2007). Bicarbonate loading to enhance training and competitive performance. Int J Sports Physiol Perform, 2, 93-7.

Cairns, S. P. (2006). Lactic acid and exercise performance : culprit or friend? Sports Med, 36, 279-91.

Cooper, R., Naclerio, F., Allgrove, J. \& Jimenez, A. (2012). Creatine supplementation with specific view to exercise/sports performance: an update. J Int Soc Sports Nutr, 9, 33.

Douroudos, Ii, Fatouros, I. G., Gourgoulis, V., Jamurtas, A. Z., Tsitsios, T., Hatzinikolaou, A., Margonis, K., Mavromatidis, K. \& Taxildaris, K. (2006). Dose-related effects of prolonged $\mathrm{NaHCO} 3$ ingestion during high-intensity exercise. Med Sci Sports Exerc, 38, 1746-53.

Febbraio, M. A., Flanagan, T. R., Snow, R. J., Zhao, S. \& Carey, M. F. (1995). Effect of creatine supplementation on intramuscular $\mathrm{TCr}$, metabolism and performance during intermittent, supramaximal exercise in humans. Acta Physiol Scand, 155, 387-95.

Goldberg, A. L., Etlinger, J. D., Goldspink, D. F. \& Jablecki, C. (1975). Mechanism of work-induced hypertrophy of skeletal muscle. Med Sci Sports, 7, 185-98.

Green, A., Hultman, E., Macdonald, I., Sewell, D. \& Greenhaff, P. (1996). Carbohydrate ingestion augments skeletal muscle creatine accumulation during creatine supplementation in humans. American Journal of PhysiologyEndocrinology And Metabolism, 271, E821-E826.

Greenhaff, P. L., Bodin, K., Soderlund, K. \& Hultman, E. (1994). Effect of oral creatine supplementation on skeletal muscle phosphocreatine resynthesis. Am J Physiol, 266, E725-30.

Griffen, C., Rogerson, D., Ranchordas, M. \& Ruddock, A. (2015). Effects of Creatine and Sodium Bicarbonate Coingestion on Multiple Indices of Mechanical Power Output During Repeated Wingate Tests in Trained Men. Int J Sport Nutr Exerc Metab, 25, 298-306.

Hultman, E. \& Sahlin, K. (1980). Acid-base balance during exercise. Exerc Sport Sci Rev, 8, 41-128.

Hultman, E., Soderlund, K., Timmons, J. A., Cederblad, G. \& Greenhaff, P. L. (1996). Muscle creatine loading in men. J Appl Physiol (1985), 81, 232-7.

Kraemer, W. J., Hakkinen, K., Newton, R. U., Mccormick, M., Nindl, B. C., Volek, J. S., Gotshalk, L. A., Fleck, S. J., Campbell, W. W., Gordon, S. E., Farrell, P. A. \& Evans, W. J. (1998). Acute hormonal responses to heavy resistance exercise in younger and older men. Eur J Appl Physiol Occup Physiol, 77, 206-11.

Lambert, C. P. \& Flynn, M. G. (2002). Fatigue during high-intensity intermittent exercise. Sports medicine, 32, 511-522.

Maccormick, V. M., Hill, L. M., Macneil, L., Burke, D. G. \& Smith-Palmer, T. (2004). Elevation of creatine in red blood cells in vegetarians and nonvegetarians after creatine supplementation. Can J Appl Physiol, 29, 704-13.

Mccaulley, G. O., Mcbride, J. M., Cormie, P., Hudson, M. B., Nuzzo, J. L., Quindry, J. C. \& Travis Triplett, N. (2009). Acute hormonal and neuromuscular responses to hypertrophy, strength and power type resistance exercise. Eur J Appl Physiol, 105, 695-704. 
Mcnaughton, L., Backx, K., Palmer, G. \& Strange, N. (1999). Effects of chronic bicarbonate ingestion on the performance of high-intensity work. Eur $J$ Appl Physiol Occup Physiol, 80, 333-6.

Mcnaughton, L. R., Siegler, J. \& Midgley, A. (2008). Ergogenic effects of sodium bicarbonate. Curr Sports Med Rep, 7, 230-6.

Mero, A. A., Kreskinen, K. L., Malvela, M. T. \& Sallinen, J. M. (2004). Combined creatine and sodium bicarbonate supplementation enhances interval swimming. Journal of Strength \& Conditioning Research, 18, 306-310.

Moritani, T. \& Devries, H. A. (1979). Neural factors versus hypertrophy in the time course of muscle strength gain. Am J Phys Med, 58, 115-30.

Nicholson, G., Mcloughlin, G., Bissas, A. \& Ispoglou, T. (2014). Do the acute biochemical and neuromuscular responses justify the classification of strengthand hypertrophy-type resistance exercise? J Strength Cond Res, 28, 3188-99.

Parkhouse, W. S. \& Mckenzie, D. C. (1984). Possible contribution of skeletal muscle buffers to enhanced anaerobic performance: a brief review. Med Sci Sports Exerc, $16,328-38$.

Requena, B., Zabala, M., Padial, P. \& Feriche, B. (2005). Sodium bicarbonate and sodium citrate: ergogenic aids? J Strength Cond Res, 19, 213-24.

Rodriguez, N., Dimarco, N., Langley, S., Association, A. D. \& Medicine, A. C. O. S. (2009). Position of the American Dietetic Association, Dietitians of Canada, and the American College of Sports Medicine: Nutrition and athletic performance. Journal of the American Dietetic Association, 109, 509.

Roza, A. M. \& Shizgal, H. M. (1984). The Harris Benedict equation reevaluated: resting energy requirements and the body cell mass. American Journal of Clinical Nutrition, 40, 168-82.

Sahlin, K. (2014). Muscle energetics during explosive activities and potential effects of nutrition and training. Sports Med, 44 Suppl 2, S167-73.

Schoenfeld, B. J. (2010). The mechanisms of muscle hypertrophy and their application to resistance training. J Strength Cond Res, 24, 2857-72.

Schoenfeld, B. J. (2013). Potential mechanisms for a role of metabolic stress in hypertrophic adaptations to resistance training. Sports Med, 43, 179-94.

Sharp, R. L., Costill, D. L., Fink, W. J. \& King, D. S. (1986). Effects of eight weeks of bicycle ergometer sprint training on human muscle buffer capacity. Int J Sports Med, 7, 13-7.

Shomrat, A., Weinstein, Y. \& Katz, A. (2000). Effect of creatine feeding on maximal exercise performance in vegetarians. Eur J Appl Physiol, 82, 321-5.

Steenge, G., Lambourne, J., Casey, A., Macdonald, I. \& Greenhaff, P. (1998). Stimulatory effect of insulin on creatine accumulation in human skeletal muscle. American Journal of Physiology-Endocrinology And Metabolism, 275, E974E979.

Steenge, G., Simpson, E. \& Greenhaff, P. (2000). Protein-and carbohydrate-induced augmentation of whole body creatine retention in humans. Journal of Applied Physiology, 89, 1165-1171.

Van Montfoort, M. C., Van Dieren, L., Hopkins, W. G. \& Shearman, J. P. (2004). Effects of ingestion of bicarbonate, citrate, lactate, and chloride on sprint running. Med Sci Sports Exerc, 36, 1239-43.

Webster, M. J., Webster, M. N., Crawford, R. E. \& Gladden, L. B. (1993). Effect of sodium bicarbonate ingestion on exhaustive resistance exercise performance. Med Sci Sports Exerc, 25, 960-5.

Winwood, P. W., Keogh, J. W. \& Harris, N. K. (2011). The strength and conditioning practices of strongman competitors. J Strength Cond Res, 25, 3118-28. 
